\title{
NORMALITY AND CONVEXITY OF THE YIELD SURFACE IN NONLINEAR PLASTICITY
}

\author{
BY \\ ROGER FOSDICK AND ERIC VOLKMANN \\ University of Minnesota, Minneapolis, Minnesota
}

\begin{abstract}
An elementary proof of the normality condition is given. In addition, the convexity-concavity character of the yield surface is found to be related to the changes in elastic stiffness that take place during plastic loading.
\end{abstract}

1. Introduction. In 1965-66, Green and Naghdi [1, 2] introduced a thermodynamic nonlinear theory of plasticity which has been the subject of much refinement and critical review over the past quarter century. A summary of the advances made in the related purely mechanical theory has been given by Casey and Naghdi [3, 4]. Both the thermodynamical and the mechanical theory have a certain appeal because of their generality, objectivism, and rational structure. As the original ideas of Green and Naghdi were advanced, Naghdi and Trapp [5] found, in the purely mechanical theory, that a postulate which restricts the work expended to be nonnegative in a cyclic strain path was sufficient to imply a certain normality condition. Roughly, the part of the stress rate that is due to the generation of plastic strain and work hardening during plastic loading must be normal to the yield surface (in strain space) and inwardly directed. In addition, by use of the same work postulate, Naghdi and Trapp [6] found that the yield surface (in both stress and strain space) must be convex, but only for a very special class of elastic-plastic materials. Essentially, this class requires the stress to depend linearly on the difference between the strain and the plastic strain, via a constant linear transformation. More recent simplified proofs of normality and restricted convexity have been given by Casey [7]. However, while Casey's idea of proof is sound and reasonably convincing, we find that the calculations that support his argument for normality are incomplete in a fundamental way.

In Sec. 3 of the present paper we give an elementary proof of normality which utilizes the above-mentioned work postulate and relies mainly on the mean value theorem of integral calculus. A brief critique of Casey's proof of normality is contained in a footnote in this section.

In Sec. 4, we consider the question of convexity of the yield surface for general elastic-plastic materials. We show that if the material is such that the local elasticity tensor (i.e., the second derivative of the stored energy function with respect to strain) 
undergoes a negative semidefinite change during plastic loading, due to the generation of plastic strain and work hardening, then the yield surface must be convex. Roughly speaking, this corresponds to a material that would become less or equally stiff with respect to future elastic unloading and reloading as a consequence of previous plastic loading. If the change in the local elasticity tensor is positive definite, then we find an inequality that places a restriction on the convexity-concavity character of the yield surface, but the restriction is not sufficiently strong to eliminate the possibility of the yield surface being concave.

2. Fundamental constitutive structure. Let $\mathbf{X}$ denote the point occupied by a particle of a body $\mathscr{B} \subset \mathbb{R}^{3}$ in a fixed reference configuration at time $t=0$, and let $\mathbf{x}=\chi(\mathbf{X}, t)$ be the position of that particle at time $t$. The deformation gradient field $\mathbf{F}(\mathbf{X}, t)$ is given by

$$
\mathbf{F} \equiv \nabla \chi,
$$

and we assume that $\operatorname{det} \mathbf{F}>0$ for all $\mathbf{X} \in \mathscr{B}$ and for all time $t$. In the purely mechanical nonlinear theory of plasticity it is common to assume the existence of a symmetric plastic strain tensor field $\mathbf{P}(\mathbf{X}, t) \in$ Sym and a work-hardening scalar field $\kappa(\mathbf{X}, t)$, and suppose that the second (symmetric) Piola-Kirchhoff stress tensor field $\boldsymbol{\tau}(\mathbf{X}, t) \in$ Sym is given by a differentiable constitutive response function through the relation

$$
\boldsymbol{\tau}=\hat{\boldsymbol{\tau}}(\mathbf{E}, \mathbf{P}, \kappa)
$$

Here,

$$
\mathbf{E} \equiv \frac{1}{2}\left(\mathbf{F}^{\mathrm{T}} \mathbf{F}-\mathbf{1}\right)
$$

is the Green-St. Venant strain tensor. In addition, a smooth yield function $g(\mathbf{E}, \mathbf{P}, \kappa)$ is introduced along with the idea that the set of all symmetric tensors

$$
\{\mathbf{E} \in \operatorname{Sym} \mid g(\mathbf{E}, \mathbf{P}, \kappa)<0\}
$$

is homeomorphic to an open ball in Sym and, for fixed $\mathbf{P}$ and $\kappa$, characterizes the region of elastic strains. The yield surface corresponding to fixed $\mathbf{P}$ and $\kappa$ is the boundary of the region of elastic strains and is given by the set of all $\mathbf{E} \in$ Sym such that

$$
g(\mathbf{E}, \mathbf{P}, \kappa)=0 .
$$

A particle $\mathbf{X} \in \mathscr{B}$ is elastic at time $t$ if

$$
g(\mathbf{E}(\mathbf{X}, t), \mathbf{P}(\mathbf{X}, t), \kappa(\mathbf{X}, t))<0 .
$$

It is only when

$$
g(\mathbf{E}(\mathbf{X}, t), \mathbf{P}(\mathbf{X}, t), \kappa(\mathbf{X}, t))=0
$$

that the particle $\mathbf{X} \in \mathscr{B}$ is said to be plastic, and then the following three alternatives characterize the loading condition for $\mathbf{X}$ :

(i) Plastic unloading: $\quad \frac{\partial g}{\partial \mathbf{E}}(\mathbf{E}(\mathbf{X}, t), \mathbf{P}(\mathbf{X}, t), \kappa(\mathbf{X}, t)) \cdot \dot{\mathbf{E}}(\mathbf{X}, t)<0$;

(ii) Plastic neutral loading: $\frac{\partial g}{\partial \mathbf{E}}(\mathbf{E}(\mathbf{X}, t), \mathbf{P}(\mathbf{X}, t), \kappa(\mathbf{X}, t)) \cdot \dot{\mathbf{E}}(\mathbf{X}, t)=0$; 
(iii) Plastic loading: $\frac{\partial g}{\partial \mathbf{E}}(\mathbf{E}(\mathbf{X}, t), \mathbf{P}(\mathbf{X}, t), \kappa(\mathbf{X}, t)) \cdot \dot{\mathbf{E}}(\mathbf{X}, t)>0$.

Whenever $\mathbf{X} \in \mathscr{B}$ is subject to plastic loading for an interval of time, it is assumed that $\mathbf{X}$ remains plastic and, therefore, that (6) holds identically. Thus,

$$
\dot{g}=\frac{\partial g}{\partial \mathbf{E}} \cdot \dot{\mathbf{E}}+\frac{\partial g}{\partial \mathbf{P}} \cdot \dot{\mathbf{P}}+\frac{\partial g}{\partial \kappa} \cdot \dot{\kappa}=0,
$$

during plastic loading.

It is assumed that plastic strain is generated only during plastic loading. Thus, it is common to postulate ${ }^{1}$ that

$$
\dot{\mathbf{P}}= \begin{cases}\mathbb{M}[\dot{\mathbf{E}}] & \text { during plastic loading } \\ 0 & \text { otherwise }\end{cases}
$$

where the fourth-order tensor $\mathbb{M}$ is given by a constitutive assumption

$$
\mathbb{M}=\widehat{\mathbb{M}}(\mathbf{E}, \mathbf{P}, \kappa) .
$$

It follows from the continuity of the right-hand side in (8) that there exists a symmetric tensor $\boldsymbol{\Sigma} \in$ Sym with

$$
\boldsymbol{\Sigma}=\widehat{\boldsymbol{\Sigma}}(\mathbf{E}, \mathbf{P}, \kappa),
$$

such that (8) may be written equivalently as

$$
\dot{\mathbf{P}}= \begin{cases}\boldsymbol{\Sigma}\left(\frac{\partial g}{\partial \mathbf{E}} \cdot \dot{\mathbf{E}}\right) & \text { during plastic loading } \\ 0 & \text { otherwise. }\end{cases}
$$

Finally, in plasticity theory it is common to assume that the evolution of hardening is governed by a differential equation of the form

$$
\dot{\kappa}=\mathbf{K} \cdot \dot{\mathbf{P}},
$$

where the symmetric tensor $\mathbf{K} \in \mathrm{Sym}$ is given by a constitutive assumption

$$
\mathbf{K}=\widehat{\mathbf{K}}(\mathbf{E}, \mathbf{P}, \kappa) \text {. }
$$

Thus, the constitutive structure of the purely mechanical theory is dependent upon the specification of the constitutive response functions, $\hat{\boldsymbol{\tau}}(\mathbf{E}, \mathbf{P}, \kappa)$ of $(2), \widehat{\boldsymbol{\Sigma}}(\mathbf{E}, \mathbf{P}, \kappa)$ of (10), and $\widehat{\mathbf{K}}(\mathbf{E}, \mathbf{P}, \kappa)$ of (13). In addition, the yield function $g(\mathbf{E}, \mathbf{P}, \kappa)$ must be specified. During plastic loading, it readily follows from (7), (11), and (12) that

$$
1+\left(\frac{\partial g}{\partial \mathbf{P}}+\frac{\partial g}{\partial \kappa} \mathbf{K}\right) \cdot \Sigma=0
$$

This constitutes a restriction of the constitutive functions $\widehat{\Sigma}(\mathbf{E}, \mathbf{P}, \kappa)$ of (10) and $\widehat{\mathbf{K}}(\mathbf{E}, \mathbf{P}, \kappa)$ of $(13)$.

\footnotetext{
${ }^{1}$ Relative to an orthonormal base $\left\{\mathbf{i}_{i}\right\}, \mathbb{M}$ has the indicial form $\mathbb{M}_{i j k l}$ and satisfies the symmetry conditions $\mathbb{M}_{i j k l}=\mathbb{M}_{j i k l}=\mathbb{M}_{i j l k}$. In addition, note that the bracket notation $\mathbb{M}[\mathbf{A}]$ has the indicial form $\mathbf{M}_{i j k l} A_{k l}$.
} 
3. The work postulate and normality condition. According to Gurtin [8, p. 185], the work is nonnegative in "closed processes" if and only if, for any time interval $\left[t_{0}, t_{1}\right]$ and any $\mathbf{X} \in \mathscr{B}$, we have

$$
\int_{t_{0}}^{t_{1}} \boldsymbol{\tau}(\mathbf{X}, t) \cdot \dot{\mathbf{E}}(\mathbf{X}, t) d t \geq 0
$$

in any process such that

$$
\chi\left(\mathbf{X}, t_{0}\right)=\chi\left(\mathbf{X}, t_{1}\right), \quad \dot{\chi}\left(\mathbf{X}, t_{0}\right)=\dot{\chi}\left(\mathbf{X}, t_{1}\right) .
$$

Here, we have used the form $\boldsymbol{\tau} \cdot \dot{\mathbf{E}}$ for the stress power per unit volume in $\mathscr{B}$ rather than the equivalent expression $\mathbf{S}^{\mathrm{T}} \cdot \dot{\mathbf{F}}$ which involves the first Piola-Kirchhoff stress tensor $\mathbf{S} \equiv \boldsymbol{\tau} \mathbf{F}^{\mathrm{T}}$. During such a process, $\boldsymbol{\tau}(\mathbf{X}, t)$ is to be computed according to (2) so that

$$
\tau(\mathbf{X}, t)=\hat{\tau}(\mathbf{E}(\mathbf{X}, t), \mathbf{P}(\mathbf{X}, t), \kappa(\mathbf{X}, t)),
$$

where $\mathbf{P}(\mathbf{X}, t)$ and $\kappa(\mathbf{X}, t)$ must evolve in agreement with (10)-(13).

Naghdi and Trapp [5] and, more recently, Casey [7] have applied the above work assumption to show that a certain second-order tensor (20), which depends on the constitutive functions $\hat{\boldsymbol{\tau}}(\mathbf{E}, \mathbf{P}, \kappa), \widehat{\boldsymbol{\Sigma}}(\mathbf{E}, \mathbf{P}, \kappa)$, and $\widehat{\mathbf{K}}(\mathbf{E}, \mathbf{P}, \kappa)$, must be normal to, and directed inward from, the yield surface. While Casey's work very much simplifies the original proof of Naghdi and Trapp, the calculations given in support of his conclusion are, in fact, not entirely correct. Nevertheless, his main idea is unequivocal. $^{2}$ Our proof of this important normality condition, which follows, is reasonably elementary and relies on the mean value theorem of integral calculus and the differentiability of the stress constitutive function $\hat{\boldsymbol{\tau}}(\mathbf{E}, \mathbf{P}, \kappa)$. It is of preliminary significance to note that, according to a result of Gurtin [8, p. 188], we may fix $\mathbf{X} \in \mathscr{B}$ and apply the work inequality (15) to any piecewise smooth closed curve $\mathbf{E}(\mathbf{X}, \cdot):\left[t_{0}, t_{1}\right] \rightarrow$ Sym that satisfies

$$
\mathbf{E}\left(\mathbf{X}, t_{0}\right)=\mathbf{E}\left(\mathbf{X}, t_{1}\right) .
$$

That is, we may drop the condition $\dot{\mathbf{E}}\left(\mathbf{X}, t_{0}\right)=\dot{\mathbf{E}}\left(\mathbf{X}, t_{1}\right)$ without affecting the validity of (15). We shall do this, but first observe, from (2), (11), and (12), that the stress rate is given by

$$
\dot{\boldsymbol{\tau}}= \begin{cases}\mathbb{C}[\dot{\mathbf{E}}]+\left(\frac{\partial g}{\partial \mathbf{E}} \cdot \dot{\mathbf{E}}\right) \boldsymbol{\sigma} & \text { during plastic loading, } \\ \mathbb{C}[\dot{\mathbf{E}}] & \text { otherwise, }\end{cases}
$$

\footnotetext{
${ }^{2}$ To briefly describe the issues here, first it is important to note that the integral defining $H_{2}(\varepsilon)$ in $(3.5)$ of Casey's paper [7, p. 66] is over the time interval of elastic behavior $(\varepsilon, 2 \varepsilon)$, which immediately follows the plastic loading part of his strain cycle. Therefore, for all time $t$ in the interval $(\varepsilon, 2 \varepsilon)$ the plastic strain $\mathbf{E}^{p}(t)$ and the strain-hardening parameter $\kappa(t)$ are both constant and equal to $\mathbf{E}^{p}(\varepsilon)$ and $\kappa(\varepsilon)$, respectively. These are the values that were generated by the end of the time interval of plastic loading $(0, \varepsilon)$. This means that the function $h(t)$ that occurs in the integrand of $H_{2}(\varepsilon)$ in Casey's work actually depends on $\varepsilon$. Since this was not recognized, the derivatives of $H_{2}(\varepsilon)$ given in (3.8) of [7] do not reflect this dependence. However, when this dependence is taken into account, notwithstanding the longer calculations, at $\varepsilon=0$ his conclusions (3.9) still hold. Thus, Casey's main idea is indifferent to the $\varepsilon$ dependency noted here, and essentially his method of proof of normality still works.

A similar observation also applies to the function $H_{2}(\varepsilon)$ in $(4.16 a)$, and its derivative in (4.24), of Casey and Tseng [9].
} 
where the local elasticity tensor $\mathbb{C}$ is defined by ${ }^{3}$

$$
\mathbb{C} \equiv \frac{\partial \hat{\boldsymbol{\tau}}}{\partial \mathbf{E}}=\widehat{\mathbb{C}}(\mathbf{E}, \mathbf{P}, \kappa)
$$

and

$$
\boldsymbol{\sigma} \equiv\left(\frac{\partial \hat{\boldsymbol{\tau}}}{\partial \mathbf{P}}+\frac{\partial \hat{\boldsymbol{\tau}}}{\partial \kappa} \otimes \mathbf{K}\right)[\boldsymbol{\Sigma}]=\hat{\boldsymbol{\sigma}}(\mathbf{E}, \mathbf{P}, \kappa) .
$$

The normality condition, to which we now turn, may be stated as follows: There exists $\gamma=\hat{\gamma}(\mathbf{E}, \mathbf{P}, \kappa) \geq 0$ such that

$$
\boldsymbol{\sigma}=-\gamma \frac{\partial g}{\partial \mathbf{E}} .
$$

First, fix $\mathbf{X} \in \mathscr{B}$ and consider its presence as implicit in the remainder of this work. Then, the work postulate corresponding to (15) may be written as

$$
\int_{t_{0}}^{t_{1}} \hat{\boldsymbol{\tau}}(\mathbf{E}(t), \mathbf{P}(t), \kappa(t)) \cdot \dot{\mathbf{E}}(t) d t \geq 0
$$

for any piecewise smooth closed curve $\mathbf{E}(\cdot):\left[t_{0}, t_{1}\right] \rightarrow \operatorname{Sym}, \mathbf{E}\left(t_{0}\right)=\mathbf{E}\left(t_{1}\right)$. Along this curve, the evolution of $\mathbf{P}(t)$ and $\kappa(t)$ is given by (10)-(13), i.e.,

$$
\dot{\mathbf{P}}(t)=\left\{\begin{array}{l}
\widehat{\boldsymbol{\Sigma}}(\mathbf{E}(t), \mathbf{P}(t), \kappa(t))\left(\frac{\partial g}{\partial \mathbf{E}}(\mathbf{E}(t), \mathbf{P}(t), \kappa(t)) \cdot \dot{\mathbf{E}}(t)\right) \\
0,
\end{array}\right.
$$

and

$$
\dot{\kappa}(t)=\widehat{\mathbf{K}}(\mathbf{E}(t), \mathbf{P}(t), \kappa(t)) \cdot \dot{\mathbf{P}}(t) .
$$

The alternative choice in (23) depends, respectively, on whether or not the condition of plastic loading is satisfied. Clearly, by considering the work postulate for closed curves which involve no plastic loading, it follows by the standard argument from elasticity theory (see, e.g., Gurtin [18, Sec. 28]) that there is a scalar function

$$
W=\widehat{W}(\mathbf{E}, \mathbf{P}, \kappa)
$$

such that

$$
\boldsymbol{\tau}=\frac{\partial \widehat{W}}{\partial \mathbf{E}} .
$$

This so-called stored energy function per unit volume in $\mathscr{B}$ determines the stress constitutive function $\hat{\boldsymbol{\tau}}(\mathbf{E}, \mathbf{P}, \kappa)$ and considerably reduces the total number of independent constitutive response functions in the theory.

Now, to show the normality condition (21), suppose that $\left(\mathbf{E}_{0}, \mathbf{P}_{0}, \kappa_{0}\right)$ satisfies $g\left(\mathbf{E}_{0}, \mathbf{P}_{0}, \kappa_{0}\right)=0$, so that $\mathbf{E}_{0} \in$ Sym is a point on the yield surface corresponding to $\left(\mathbf{P}_{0}, \kappa_{0}\right)$. Consider the piecewise smooth closed curve $\mathbf{E}(\cdot):\left[t_{0}, t_{1}\right] \rightarrow$ Sym defined by

$$
\mathbf{E}(t)= \begin{cases}\mathbf{E}_{0}+\left(\frac{t-t_{0}}{t^{*}-t_{0}}\right) \varepsilon \mathbf{M}, & t \in\left[t_{0}, t^{*}\right], \\ \mathbf{E}_{0}+\left(\frac{t_{1}-t}{t_{1}-t^{*}}\right) \varepsilon \mathbf{M}, & t \in\left[t^{*}, t_{1}\right],\end{cases}
$$

\footnotetext{
${ }^{3}$ Relative to an orthonormal basis $\left[i_{i}\right], \mathbb{C}$ has the indicial form $\mathbb{C}_{i j k l}$ and satisfies the symmetry condi-
} tions $\mathbb{C}_{i j k l}=\mathbb{C}_{j i k l}=\mathbb{C}_{k l i j}$. 
where $\varepsilon>0$, and $\mathbf{M} \in \operatorname{Sym}$ is any tensor such that $|\mathbf{M}|=1$ and

$$
\frac{\partial g}{\partial \mathbf{E}}\left(\mathbf{E}_{0}, \mathbf{P}_{0}, \kappa_{0}\right) \cdot \mathbf{M}>0 .
$$

During the time interval $\left[t_{0}, t^{*}\right], t_{0}<t^{*}<t_{1}$, plastic loading takes place, and in the remainder of this strain cycle, $t \in\left[t^{*}, t_{1}\right]$, the condition of plastic unloading applies. Thus, for $t \in\left(t_{0}, t^{*}\right)$ we have

$$
\begin{gathered}
\left(t^{*}-t_{0}\right) \dot{\mathbf{P}}(t)=\widehat{\boldsymbol{\Sigma}}(\mathbf{E}(t), \mathbf{P}(t), \kappa(t))\left(\frac{\partial g}{\partial \mathbf{E}}(\mathbf{E}(t), \mathbf{P}(t), \kappa(t)) \cdot \varepsilon \mathbf{M}\right), \\
\dot{\kappa}(t)=\widehat{\mathbf{K}}(\mathbf{E}(t), \mathbf{P}(t), \kappa(t)) \cdot \dot{\mathbf{P}}(t),
\end{gathered}
$$

where $\mathbf{E}(t)$ is given by $(27)_{1}$. Notice that

$$
\mathbf{P}\left(t_{0}\right)=\mathbf{P}_{0}, \quad \kappa\left(t_{0}\right)=\kappa_{0} .
$$

For $t \in\left(t^{*}, t_{1}\right)$ the condition of plastic unloading requires that $\dot{\mathbf{P}}(t)=0$ and $\dot{\kappa}(t)=$ 0 , so during this time interval we have

$$
\mathbf{P}(t)=\mathbf{P}\left(t^{*}\right), \quad \kappa(t)=\kappa\left(t^{*}\right), \quad t \in\left(t^{*}, t_{1}\right) .
$$

Thus, (22) now has the more specific form

$$
\begin{aligned}
\int_{t_{0}}^{t^{*}} & \hat{\boldsymbol{\tau}} \\
& \left(\mathbf{E}_{0}+\left(\frac{t-t_{0}}{t^{*}-t_{0}}\right) \varepsilon \mathbf{M}, \mathbf{P}(t), \kappa(t)\right) \cdot \varepsilon \mathbf{M} d t /\left(t^{*}-t_{0}\right) \\
& -\int_{t^{*}}^{t_{1}} \hat{\boldsymbol{\tau}}\left(\mathbf{E}_{0}+\left(\frac{t_{1}-t}{t_{1}-t^{*}}\right) \varepsilon \mathbf{M}, \mathbf{P}\left(t^{*}\right), \kappa\left(t^{*}\right)\right) \cdot \varepsilon \mathbf{M} d t /\left(t_{1}-t^{*}\right) \geq 0,
\end{aligned}
$$

where $\mathbf{P}(t)$ and $\kappa(t)$, for $t \in\left(t_{0}, t^{*}\right]$, are generated by (29).

Now, fix the units in which time is measured and let $\left[t_{0}, t_{1}\right]$ and $\left[t_{0}, t^{*}\right]$ be such that

$$
t_{1}-t_{0}=\varepsilon, \quad t^{*}-t_{0}=\lambda \varepsilon,
$$

where $\lambda \in(0,1)$. If we then change the integration variable in the first integral above so that $t \mapsto \alpha=t-t_{0}$, and in the second integral so that $t \mapsto \alpha=$ $\lambda\left(t_{1}-t\right) /(1-\lambda)$, the inequality may be readily reduced to the more convenient form

$$
\begin{aligned}
\frac{1}{\lambda} \int_{0}^{\lambda \varepsilon}[\hat{\boldsymbol{\tau}} & \left(\mathbf{E}_{0}+\frac{\alpha}{\lambda} \mathbf{M}, \overline{\mathbf{P}}(\alpha), \bar{\kappa}(\alpha)\right) \\
& \left.-\hat{\boldsymbol{\tau}}\left(\mathbf{E}_{0}+\frac{\alpha}{\lambda} \mathbf{M}, \overline{\mathbf{P}}(\lambda \varepsilon), \bar{\kappa}(\lambda \varepsilon)\right)\right] \cdot \mathbf{M} d \alpha \geq 0 .
\end{aligned}
$$

Here, the functions $\overline{\mathbf{P}}(\cdot)$ and $\bar{\kappa}(\cdot)$ are defined by $\overline{\mathbf{P}}(\alpha) \equiv \mathbf{P}\left(t_{0}+\alpha\right), \bar{\kappa}(\alpha)=\kappa\left(t_{0}+\alpha\right)$, and, because of (30) and (31), they have the properties

$$
\overline{\mathbf{P}}(0)=\mathbf{P}_{0}, \quad \bar{\kappa}(0)=\kappa_{0} .
$$

Notice that, by (29), we have the following two additional properties:

$$
\begin{aligned}
\lambda \frac{d}{d \alpha} \overline{\mathbf{P}}(\alpha)= & \widehat{\boldsymbol{\Sigma}}\left(\mathbf{E}_{0}+\frac{\alpha}{\lambda} \mathbf{M}, \overline{\mathbf{P}}(\alpha), \bar{\kappa}(\alpha)\right) \\
& \times\left(\frac{\partial g}{\partial \mathbf{E}}\left(\mathbf{E}_{0}+\frac{\alpha}{\lambda} \mathbf{M}, \overline{\mathbf{P}}(\alpha), \bar{\kappa}(\alpha)\right) \cdot \mathbf{M}\right), \\
\frac{d}{d \alpha} \bar{\kappa}(\alpha)= & \widehat{\mathbf{K}}\left(\mathbf{E}_{0}+\frac{\alpha}{\lambda} \mathbf{M}, \overline{\mathbf{P}}(\alpha), \bar{\kappa}(\alpha)\right) \cdot \frac{d}{d \alpha} \overline{\mathbf{P}}(\alpha) .
\end{aligned}
$$


Finally, observe that the mean value theorem allows us to write (33) in the equivalent form

$$
\varepsilon\left[\hat{\boldsymbol{\tau}}\left(\mathbf{E}_{0}+\frac{\lambda_{0}}{\lambda} \varepsilon \mathbf{M}, \overline{\mathbf{P}}\left(\lambda_{0} \varepsilon\right), \bar{\kappa}\left(\lambda_{0} \varepsilon\right)\right)-\hat{\boldsymbol{\tau}}\left(\mathbf{E}_{0}+\frac{\lambda_{0}}{\lambda} \varepsilon \mathbf{M}, \overline{\mathbf{P}}(\lambda \varepsilon), \bar{\kappa}(\lambda \varepsilon)\right)\right] \cdot \mathbf{M} \geq 0,
$$

where $\lambda_{0}$ is some number in the interval $(0, \lambda)$.

It is now straightforward to use the differentiability of $\hat{\boldsymbol{\tau}}(\mathbf{E}, \mathbf{P}, \kappa)$, along with (35) and (20), to rewrite $(36)$ as

$$
\varepsilon^{2}\left(\lambda_{0}-\lambda\right) \frac{\partial g}{\partial \mathbf{E}}\left(\mathbf{E}_{0}, \mathbf{P}_{0}, \kappa_{0}\right) \cdot \mathbf{M}\left(\hat{\boldsymbol{\sigma}}\left(\mathbf{E}_{0}, \mathbf{P}_{0}, \kappa_{0}\right) \cdot \mathbf{M}\right)+O\left(\varepsilon^{3}\right) \geq 0 .
$$

Thus, by (28) and the fact that $\lambda_{0}<\lambda$, we may conclude that $\hat{\boldsymbol{\sigma}}\left(\mathbf{E}_{0}, \mathbf{P}_{0}, \kappa_{0}\right) \cdot \mathbf{M} \leq 0$ for all $\mathbf{M} \in$ Sym such that

$$
\frac{\partial g}{\partial \mathbf{E}}\left(\mathbf{E}_{0}, \mathbf{P}_{0}, \kappa_{0}\right) \cdot \mathbf{M}>0 .
$$

According to an argument of Naghdi and Trapp [5], this implies that there exists $\gamma=\hat{\gamma}\left(\mathbf{E}_{0}, \mathbf{P}_{0}, \kappa_{0}\right) \geq 0$ such that

$$
\hat{\sigma}\left(\mathbf{E}_{0}, \mathbf{P}_{0}, \kappa_{0}\right)=-\hat{\gamma}\left(\mathbf{E}_{0}, \mathbf{P}_{0}, \kappa_{0}\right) \frac{\partial g}{\partial \mathbf{E}}\left(\mathbf{E}_{0}, \mathbf{P}_{0}, \kappa_{0}\right),
$$

which proves the normality condition $(21)$.

4. Convexity of the yield surface. Here, we give a sufficient condition, in terms of the change in the local elasticity tensor during plastic loading, for the convexity of the yield surface, and then show that if the condition is not satisfied the yield surface cannot be "too concave." In order to express this in more quantitative terms, first observe from (11), (12), and (19) that

where $^{4}$

$$
\dot{\mathbb{C}}=\left\{\begin{array}{l}
\frac{\partial \widehat{\mathbb{C}}}{\partial \mathbf{E}}[\dot{\mathbf{E}}]+\left(\frac{\partial g}{\partial \mathbf{E}} \cdot \dot{\mathbf{E}}\right) \Gamma \quad \text { during plastic loading } \\
\frac{\partial \widehat{\mathbb{C}}}{\partial \mathbf{E}}[\dot{\mathbf{E}}] \quad \text { otherwise }
\end{array}\right.
$$

$$
\boldsymbol{\Gamma} \equiv\left(\frac{\partial \widehat{\mathbb{C}}}{\partial \mathbf{P}}+\frac{\partial \widehat{\mathbb{C}}}{\partial \kappa} \otimes \mathbf{K}\right)[\Sigma]=\widehat{\Gamma}(\mathbf{E}, \mathbf{P}, \kappa)
$$

This tensor is a measure of the irreversible change that takes place in the local elasticity tensor $\mathbb{C}$ during plastic loading. It will have an effect on the convexity character of the yield surface.

Now, to facilitate our work, let $\mathbf{E}_{a} \in$ Sym be a point inside the region of elastic strains corresponding to $\left(\mathbf{P}_{0}, \kappa_{0}\right)$ so that $g\left(\mathbf{E}_{a}, \mathbf{P}_{0}, \kappa_{0}\right)<0$. Then, let $\mathbf{A}(\cdot):\left[a, t_{0}\right]$ $\rightarrow$ Sym be a smooth curve in this region of elastic strains that satisfies $\mathbf{A}(a)=\mathbf{E}_{a}$, $\mathbf{A}\left(t_{0}\right)=\mathbf{E}_{0}$, where $\mathbf{E}_{0}$ is a point on the yield surface corresponding to $\left(\mathbf{P}_{0}, \kappa_{0}\right)$. Consider the piecewise smooth closed curve $\mathbf{E}(\cdot):[a, b] \rightarrow$ Sym defined by

$$
\mathbf{E}(t)=\left\{\begin{array}{l}
\mathbf{A}(t), \quad t \in\left[a, t_{0}\right], \\
\text { given by }(27),(28) \text { for } t \in\left[t_{0}, t_{1}\right], \\
\mathbf{A}\left(t_{1}+t_{0}-t\right), \quad t \in\left[t_{1}, b\right]
\end{array}\right.
$$

\footnotetext{
${ }^{4}$ The fourth-order tensor $\Gamma$ satisfies the same symmetry properties as $\mathbb{C}$.
} 
where $b=t_{1}+t_{0}-a$. During the time interval $\left[a, t_{0}\right]$ elastic loading takes place on a strain path that is inside the region of elastic strains corresponding to $\left(\mathbf{P}_{0}, \kappa_{0}\right)$. Then, according to (27) and (28), during the time interval $\left[t_{0}, t_{1}\right]$ plastic loading is in effect until the specified intermediate time $t^{*} \in\left(t_{0}, t_{1}\right)$ and thereafter in this interval the strain path is reversed and unloading takes place inside the newly generated region of elastic strains corresponding to $\left(\mathbf{P}\left(t^{*}\right), \kappa\left(t^{*}\right)\right)$. This unloading continues during the time interval $\left[t_{1}, b\right]$ on a reversal of the strain path that was followed in the initial interval. For this closed strain curve, our considerations of the previous section, which led up to (37), allow us to write the work postulate (22) in the form

$$
I+\varepsilon^{2}\left(\lambda_{0}-\lambda\right) \frac{\partial g}{\partial \mathbf{E}}\left(\mathbf{E}_{0}, \mathbf{P}_{0}, \kappa_{0}\right) \cdot \mathbf{M}\left(\hat{\boldsymbol{\sigma}}\left(\mathbf{E}_{0}, \mathbf{P}_{0}, \kappa_{0}\right) \cdot \mathbf{M}\right)+O\left(\varepsilon^{3}\right) \geq 0,
$$

where $I$ is defined by

$$
I=\int_{a}^{t_{0}}\left(\hat{\boldsymbol{\tau}}\left(\mathbf{A}(t), \mathbf{P}_{0}, \kappa_{0}\right)-\hat{\boldsymbol{\tau}}\left(\mathbf{A}(t), \mathbf{P}\left(t^{*}\right), \kappa\left(t^{*}\right)\right)\right) \cdot \dot{\mathbf{A}}(t) d t .
$$

Here, by a change of integration variable, we have combined the two elementary work integrals that naturally appear over the intervals $\left[a, t_{0}\right]$ and $\left[t_{1}, b\right]$ into the more convenient single integral expression (42). Actually, because of (26), this integral is path independent and has the value

$$
\begin{aligned}
I= & \widehat{W}\left(\mathbf{E}_{a}, \mathbf{P}\left(t^{*}\right), \kappa\left(t^{*}\right)\right)-\widehat{W}\left(\mathbf{E}_{0}, \mathbf{P}\left(t^{*}\right), \kappa\left(t^{*}\right)\right) \\
& -\left(\widehat{W}\left(\mathbf{E}_{a}, \mathbf{P}_{0}, \kappa_{0}\right)-\widehat{W}\left(\mathbf{E}_{0}, \mathbf{P}_{0}, \kappa_{0}\right)\right) .
\end{aligned}
$$

We now recall, from (30), (32), and (34), that

$$
\begin{array}{lll}
\mathbf{P}\left(t^{*}\right)=\mathbf{P}\left(t_{0}+\lambda \varepsilon\right)=\overline{\mathbf{P}}(\lambda \varepsilon), & \overline{\mathbf{P}}(0)=\mathbf{P}_{0}, \\
\kappa\left(t^{*}\right)=\kappa\left(t_{0}+\lambda \varepsilon\right)=\bar{\kappa}(\lambda \varepsilon), & \bar{\kappa}(0)=\kappa_{0},
\end{array}
$$

and observe that for a sufficiently smooth stored energy function, (43) may be written in the form

$$
\begin{aligned}
I= & \left\{\varepsilon \hat{\boldsymbol{\sigma}}\left(\mathbf{E}_{0}, \mathbf{P}_{0}, \kappa_{0}\right)\left(\frac{\partial g}{\partial \mathbf{E}}\left(\mathbf{E}_{0}, \mathbf{P}_{0}, \kappa_{0}\right) \cdot \mathbf{M}\right)+O\left(\varepsilon^{2}\right)\right\} \cdot\left(\mathbf{E}_{a}-\mathbf{E}_{0}\right) \\
& +\left\{\frac{\varepsilon}{2} \widehat{\boldsymbol{\Gamma}}\left(\mathbf{E}_{0}, \mathbf{P}_{0}, \kappa_{0}\right)\left(\frac{\partial g}{\partial \mathbf{E}}\left(\mathbf{E}_{0}, \mathbf{P}_{0}, \kappa_{0}\right) \cdot \mathbf{M}\right)+O\left(\varepsilon^{2}\right)\right\}\left[\mathbf{E}_{a}-\mathbf{E}_{0}\right] \cdot\left(\mathbf{E}_{a}-\mathbf{E}_{0}\right) \\
& +O(\varepsilon) O\left(\left|\mathbf{E}_{a}-\mathbf{E}_{0}\right|^{3}\right) .
\end{aligned}
$$

To obtain this, we have, of course, used (26) and applied the definitions (19), (20), and (39), and we have also used (35). Otherwise, the calculation is straightforward. Now, by substituting (44) into (41), dividing the resulting inequality through by $\varepsilon$, and letting $\varepsilon \rightarrow 0$ we reach

$$
\begin{aligned}
\left(\frac{\partial g}{\partial \mathbf{E}}\left(\mathbf{E}_{0}, \mathbf{P}_{0}, \kappa_{0}\right) \cdot \mathbf{M}\right) & \left(\hat{\boldsymbol{\sigma}}\left(\mathbf{E}_{0}, \mathbf{P}_{0}, \kappa_{0}\right) \cdot\left(\mathbf{E}_{a}-\mathbf{E}_{0}\right)\right. \\
+ & \left.\frac{1}{2} \widehat{\boldsymbol{\Gamma}}\left(\mathbf{E}_{0}, \mathbf{P}_{0}, \kappa_{0}\right)\left[\mathbf{E}_{a}-\mathbf{E}_{0}\right] \cdot\left(\mathbf{E}_{a}-\mathbf{E}_{0}\right)+O\left(\left|\mathbf{E}_{a}-\mathbf{E}_{0}\right|^{3}\right)\right) \geq 0
\end{aligned}
$$


for all $\mathbf{M} \in$ Sym such that (28) holds. Because of (28) we may drop the multiplying factor in front of this inequality, and then by using the normality condition (21) we have

$$
\begin{aligned}
& -\hat{\gamma}\left(\mathbf{E}_{0}, \mathbf{P}_{0}, \kappa_{0}\right) \frac{\partial g}{\partial \mathbf{E}}\left(\mathbf{E}_{0}, \mathbf{P}_{0}, \kappa_{0}\right) \cdot\left(\mathbf{E}_{a}-\mathbf{E}_{0}\right) \\
& \quad+\frac{1}{2} \widehat{\boldsymbol{\Gamma}}\left(\mathbf{E}_{0}, \mathbf{P}_{0}, \kappa_{0}\right)\left[\mathbf{E}_{a}-\mathbf{E}_{0}\right] \cdot\left(\mathbf{E}_{a}-\mathbf{E}_{0}\right)+O\left(\left|\mathbf{E}_{a}-\mathbf{E}_{0}\right|^{3}\right) \geq 0,
\end{aligned}
$$

where $\hat{\gamma}\left(\mathbf{E}_{0}, \mathbf{P}_{0}, \kappa_{0}\right) \geq 0$. We are now in a position to prove the following

Theorem. If $\widehat{\boldsymbol{\Gamma}}\left(\mathbf{E}_{0}, \mathbf{P}_{0}, \kappa_{0}\right)$ is negative semidefinite, then for $\hat{\gamma}\left(\mathbf{E}_{0}, \mathbf{P}_{0}, \kappa_{0}\right)>0$ the yield surface $g\left(\mathbf{E}, \mathbf{P}_{0}, \kappa_{0}\right)=0$ corresponding to $\left(\mathbf{P}_{0}, \kappa_{0}\right)$ is convex at $\mathbf{E}=\mathbf{E}_{0}$.

Proof. Suppose the yield surface corresponding to $\left(\mathbf{P}_{0}, \kappa_{0}\right)$ is not convex at $\mathbf{E}_{0}$. Then, in an arbitrary small neighborhood of $\mathbf{E}_{0}$, there must be a point $\mathbf{E}_{a} \in \operatorname{Sym}$ that is both inside the region of elastic strains corresponding to $\left(\mathbf{P}_{0}, \kappa_{0}\right)$ and such that

$$
\frac{\partial g}{\partial \mathbf{E}}\left(\mathbf{E}_{0}, \mathbf{P}_{0}, \kappa_{0}\right) \cdot\left(\mathbf{E}_{a}-\mathbf{E}_{0}\right)>0 .
$$

In this case, the first term in the inequality (45) is negative, and, by hypothesis, the second term is nonpositive. Thus, the sum is negative and consequently, the inequality is not satisfied for $\left|\mathbf{E}_{a}-\mathbf{E}_{0}\right|$ sufficiently small. This contradiction then allows us to conclude that the yield surface corresponding to $\left(\mathbf{P}_{0}, \kappa_{0}\right)$ must be convex at $\mathbf{E}_{0}$.

If $\widehat{\boldsymbol{\Gamma}}\left(\mathbf{E}_{0}, \mathbf{P}_{0}, \kappa_{0}\right)$ is positive definite, an argument like that in the above proof, which is based on the premise that the yield surface corresponding to $\left(\mathbf{P}_{0}, \kappa_{0}\right)$ is not convex at $\mathbf{E}_{0}$, will need to be refined. This is because the first two terms in (45) are, now, of opposite sign and also of the same order of magnitude in $\left|\mathbf{E}_{a}-\mathbf{E}_{0}\right|$, i.e., when $\left|\mathbf{E}_{a}-\mathbf{E}_{0}\right|$ is small, $\mathbf{E}_{a}-\mathbf{E}_{0}$ is also nearly orthogonal to $(\partial g / \partial \mathbf{E})\left(\mathbf{E}_{0}, \mathbf{P}_{0}, \kappa_{0}\right)$. To argue this case, then, it is first helpful to observe that in (45) we may limit $\mathbf{E}_{a}$ to be a point on the yield surface corresponding to $\left(\mathbf{P}_{0}, \kappa_{0}\right)$. Then,

$$
\begin{aligned}
0=g\left(\mathbf{E}_{a}, \mathbf{P}_{0}, \kappa_{0}\right)= & g\left(\mathbf{E}_{0}, \mathbf{P}_{0}, \kappa_{0}\right)+\frac{\partial g}{\partial \mathbf{E}}\left(\mathbf{E}_{0}, \mathbf{P}_{0}, \kappa_{0}\right) \cdot\left(\mathbf{E}_{a}-\mathbf{E}_{0}\right) \\
& +\frac{1}{2} \frac{\partial^{2} g}{\partial \mathbf{E} \partial \mathbf{E}}\left(\mathbf{E}_{0}, \mathbf{P}_{0}, \kappa_{0}\right)\left[\mathbf{E}_{a}-\mathbf{E}_{0}\right] \cdot\left(\mathbf{E}_{a}-\mathbf{E}_{0}\right)+O\left(\left|\mathbf{E}_{a}-\mathbf{E}_{0}\right|^{3}\right),
\end{aligned}
$$

where $g\left(\mathbf{E}_{0}, \mathbf{P}_{0}, \kappa_{0}\right)=0$. Using this, we find that the inequality (45) becomes

$$
\begin{aligned}
& \frac{1}{2}\left(\hat{\gamma}\left(\mathbf{E}_{0}, \mathbf{P}_{0}, \kappa_{0}\right) \frac{\partial^{2} g}{\partial \mathbf{E} \partial \mathbf{E}}\left(\mathbf{E}_{0}, \mathbf{P}_{0}, \kappa_{0}\right)+\widehat{\boldsymbol{\Gamma}}\left(\mathbf{E}_{0}, \mathbf{P}_{0}, \kappa_{0}\right)\right)\left[\mathbf{E}_{a}-\mathbf{E}_{0}\right] \cdot\left(\mathbf{E}_{a}-\mathbf{E}_{0}\right) \\
& \quad+O\left(\left|\mathbf{E}_{a}-\mathbf{E}_{0}\right|^{3}\right) \geq 0 .
\end{aligned}
$$

Thus, by dividing through by $\left|\mathbf{E}_{a}-\mathbf{E}_{0}\right|^{2}$ and then letting $\mathbf{E}_{a} \rightarrow \mathbf{E}_{0}$, with $\mathbf{E}_{a}$ always staying on the yield surface corresponding to $\left(\mathbf{P}_{0}, \kappa_{0}\right)$, we reach 


$$
\left(\hat{\gamma}\left(\mathbf{E}_{0}, \mathbf{P}_{0}, \kappa_{0}\right) \frac{\partial^{2} g}{\partial \mathbf{E} \partial \mathbf{E}}\left(\mathbf{E}_{0}, \mathbf{P}_{0}, \kappa_{0}\right)+\widehat{\boldsymbol{\Gamma}}\left(\mathbf{E}_{0}, \mathbf{P}_{0}, \kappa_{0}\right)\right)[\mathbf{N}] \cdot \mathbf{N} \geq 0
$$

for all $\mathbf{N} \in$ Sym with $|\mathbf{N}|=1$ and $(\partial g / \partial \mathbf{E})\left(\mathbf{E}_{0}, \mathbf{P}_{0}, \kappa_{0}\right) \cdot \mathbf{N}=0$. This shows that if $\widehat{\boldsymbol{\Gamma}}\left(\mathbf{E}_{0}, \mathbf{P}_{0}, \kappa_{0}\right)$ is positive definite and $\hat{\gamma}\left(\mathbf{E}_{0}, \mathbf{P}_{0}, \kappa_{0}\right)>0$, then the yield surface corresponding to $\left(\mathbf{P}_{0}, \kappa_{0}\right)$ can be, but is not necessarily, concave at $\mathbf{E}=\mathbf{E}_{0}$. While the "amount" of allowable concavity is restricted by the inequality (46), it does not seem fruitful to further pursue this quantitatively at this stage.

We turn, finally, to an interpretation of the definiteness conditions imposed on $\widehat{\boldsymbol{\Gamma}}\left(\mathbf{E}_{0}, \mathbf{P}_{0}, \kappa_{0}\right)$ in the earlier theorem and statement above for the special constitutive assumption

$$
\begin{aligned}
W & =\widehat{W}(\mathbf{E}, \mathbf{P}, \kappa) \\
& =\frac{1}{2} \widehat{\mathbb{C}}(\mathbf{P}, \kappa)[\mathbf{E}-\mathbf{P}] \cdot(\mathbf{E}-\mathbf{P})
\end{aligned}
$$

Here, $\boldsymbol{\tau}=\partial \widehat{W} / \partial \mathbf{E}=\widehat{\mathbb{C}}(\mathbf{P}, \kappa)[\mathbf{E}-\mathbf{P}]$, and $\mathbb{C}=\partial \hat{\boldsymbol{\tau}} / \partial \mathbf{E}=\widehat{\mathbb{C}}(\mathbf{P}, \kappa)$.

It is common to assume that $\mathbb{C}$ is positive definite. In this case, from (38) and (39) we get

$$
\dot{\mathbb{C}}=\left\{\begin{array}{l}
\left(\frac{\partial g}{\partial \mathbf{E}} \cdot \dot{\mathbf{E}}\right) \Gamma \quad \text { during plastic loading } \\
0 \text { otherwise }
\end{array}\right.
$$

where, it should be recalled, $\boldsymbol{\Gamma}=\widehat{\boldsymbol{\Gamma}}(\mathbf{E}, \mathbf{P}, \kappa)$ may depend on $\mathbf{E}$ as well as $\mathbf{P}$ and $\kappa$, since the constitutive functions $\widehat{\mathbf{K}}$ and $\widehat{\boldsymbol{\Sigma}}$ may exhibit this full dependency.

During plastic loading we have $(\partial g / \partial \mathbf{E}) \cdot \dot{\mathbf{E}}>0$ and, then, $\dot{\mathbb{C}}$ and $\boldsymbol{\Gamma}$ are positive or negative definite in sympathy with one another. Thus, when $\widehat{\boldsymbol{\Gamma}}\left(\mathbf{E}_{0}, \mathbf{P}_{0}, \kappa_{0}\right)$ is negative semidefinite, as required in the earlier theorem, the local elasticity tensor $\widehat{\mathbb{C}}(\mathbf{P}, \kappa)$ is instantaneously undergoing a change at $\left(\mathbf{P}_{0}, \kappa_{0}\right)$ as a result of plastic loading that tends to make the material equally or less stiff with respect to future elastic unloading or reloading. When this is the case, according to the theorem the yield surface corresponding to $\left(\mathbf{P}_{0}, \kappa_{0}\right)$ is convex at $\mathbf{E}_{0}$. Clearly, the case $\widehat{\mathbb{C}}(\mathbf{P}, \kappa)=$ constant, independent of $\mathbf{P}$ and $\kappa$, is included here as a special case, but, of course, the convexity here is well known [6,7]. On the other hand, when $\widehat{\boldsymbol{\Gamma}}\left(\mathbf{E}_{0}, \mathbf{P}_{0}, \kappa_{0}\right)$ is positive definite, the local elasticity tensor $\widehat{\mathbb{C}}(\mathbf{P}, \kappa)$ must change at $\left(\mathbf{P}_{0}, \kappa_{0}\right)$ so as to stiffen ${ }^{5}$ the response of the material to future elastic unloading and reloading. While the yield surface corresponding to $\left(\mathbf{P}_{0}, \kappa_{0}\right)$ may be convex at $\mathbf{E}_{0}$, our earlier argument shows only that there is a mild bound on its degree of concavity.

\footnotetext{
${ }^{5}$ See Fig. 1 for an interpretation of the idea of less stiff and more stiff elastic response.
} 


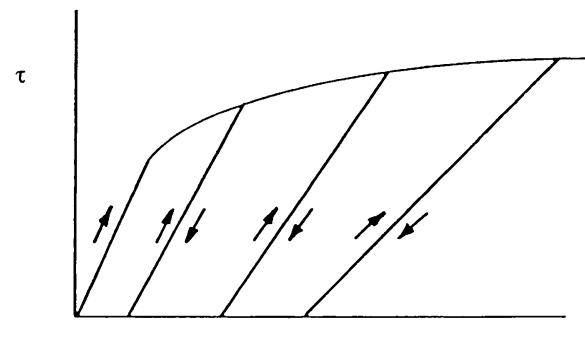

F

(a) The case $\Gamma$ is negative definite: Less stiff elastic response.

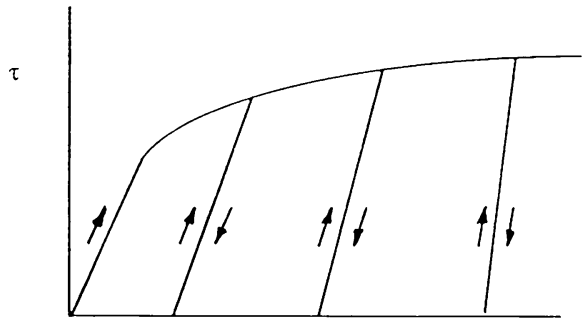

E

(b) The case $\Gamma$ is positive definite: More stiff elastic response.

Fig. 1. The effect of $\Gamma$ on stiffness for the special material of (47).

Acknowledgment. We gratefully acknowledge Alliant Techsystems Inc. for their support of this research. The partial support of the Institute for Mathematics and its Applications at the University of Minnesota is also acknowledged.

\section{REFERENCES}

[1] A. E. Green and P. M. Naghdi, A general theory of elastic-plastic continuum, Arch. Rational Mech. Anal. 18, 251-281 (1965)

[2] _ , A thermodynamic development of elastic-plastic continua, Proc. IUTAM on Irreversible Aspects of Continuum Mechanics and Transfer of Physical Characteristics in Moving Fluids (H. Parkus and L. I. Sedov, eds.), Springer-Verlag, New York, 1966, pp. 117-131

[3] J. Casey and P. M. Naghdi, Constitutive results for finitely deforming elastic-plastic materials, Constitutive Equations: Macro and Computation Aspects (K. J. William, ed.), ASME, New York, 1984, pp. 53-71

[4] _ Strain-hardening response of elastic-plastic materials, Mechanics of Materials (C. S. Desai and R. H. Gallagher, eds.), Wiley, New York, 1984, pp. 61-89

[5] P. M. Naghdi and J. A. Trapp, Restrictions on constitutive equations of finitely deformed elasticplastic materials, Quart. J. Mech. Appl. Math. 28, 25-46 (1975)

[6] _ On the nature of normality of plastic strain rate and convexity of yield surfaces in plasticity, J. Appl. Mech. 42, 61-66 (1975)

[7] J. Casey, A simple proof of a result in finite plasticity, Quart. Appl. Math. 62, 61-71 (1984)

[8] M. E. Gurtin, An introduction to continuum mechanics, Academic Press, New York, 1981

[9] J. Casey and M. Tseng, A constitutive restriction related to convexity of yield surfaces in plasticity, Z. Angew. Math. Phys. 35, 478-496 (1984) 